\title{
ApplicationForum \\ Super-Resolution Microscopy Gives New Insights into Nuclear Pore Complex Organization
}

EMBL researchers combine Ground State Depletion Microscopy with a novel image analysis method

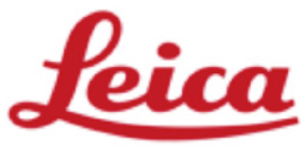

MICROSYSTEM S
The Nuclear Pore Complex (NPC) is a large protein complex in the nuclear membrane, representing the gate to the eukaryotic genetic makeup. This complex consists of several hundreds of proteins that form a selective gate for compounds destined to enter or leave the nucleus.

Because of this outstanding function, the structure of the NPC is of great interest. Structural analysis of the NPC has been limited mainly to crystallization studies or electron microscopy (EM). Several structures of single components have already been deciphered by crystallography. However, the organization of individual proteins inside the complex remained elusive. One gap has now been closed by the use of super-resolution microscopy.

Jan Ellenberg and his team of scientists at the EMBL in Heidelberg have obtained new insights into the NPC structure with the help of Ground State Depletion (GSD) microscopy.

Anna Szymborska recently published the results of this research in the Science article "Nuclear Pore Scaffold Structure Analyzed by Super-Resolution Microscopy and Particle Averaging" and comments on her achievements and the potential of Ground State Depletion microscopy for protein complex analysis in the following interview.

\section{What are the advantages of the GSDIM method for your research?}

In our research we try to understand how large multiprotein complexes, such as the nuclear pore complex (NPC), are built. Because of their size and complexity, such molecular machines are beyond the scope of a single method and have long been a challenge for structural biology. Atomic-resolution methods such as X-ray crystallography or NMR require a purified specimen and are not suitable for very large assemblies. Although, electron microscopy allows the observation of large complexes in their native environment in the cell, it is often very difficult to assign the electron density to individual proteins. In fluorescence microscopy the identity of the protein is known and super-resolution (SR) now allows us to visualize details below the diffraction limit. When SR is combined with particle averaging, proteins' positions can be mapped to a subnanometer precision, a scale that makes light microscopy applicable to structural studies of large complexes. SR microscopy can therefore link different types of data and eventually help generate pseudo-atomic models of multiprotein assemblies. Additionally, a big advantage of especially GSDIM and other localization SR methods is that they are relatively simple to use and routinely allow the resolution of proteins which are 10-20 nm apart. This is important for us because we can acquire large datasets and look at many different markers in a relatively efficient manner.
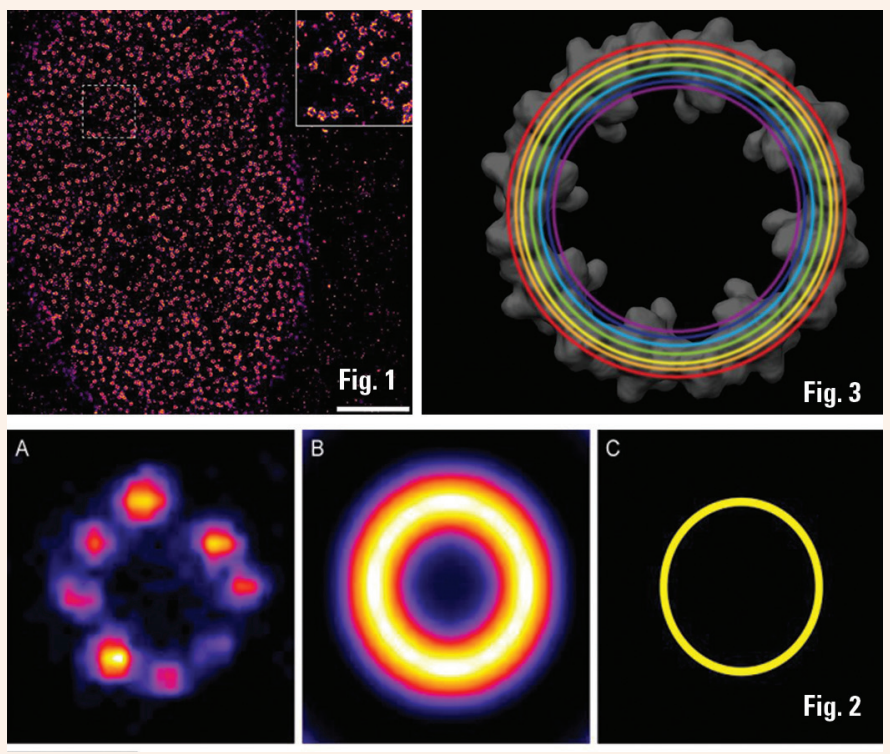

Fig. 1: The bottom surface of a nucleus of a U2OS cell labeled with an antibody against nucleoporin Nup133 and a secondary antibody conjugated to Alexa Fluor 647. Individual NPCs viewed along the transport axis are visible as ringshaped structures. Scale bar: $3 \mu \mathrm{m}$.

Fig. 2: Example results of the averaging procedure. (A) An image of an NPC labeled with an antibody against Nup96 (Gaussian filtered). (B) An average image of NPC stained with an antibody against Nup96 generated by alignment and summing of over 8,000 images of individual pores. (C) The average position of fluorescent markers on Nup96 around the center of the NPC, determined from the profile of the average image. Scale bar: $0.1 \mu \mathrm{m}$.

Fig. 3: Applying the averaging procedure to several epitopes of the Nup107160 subcomplex (depicted by different colors), their relative positions can be determined. Staining was achieved by using nanobody labeling of GFP fusion proteins. Positional information was overlaid with the EM structure of the cytoplasmic ring of the NPC.

For the full interview, visit:

http://www.leica-microsystems.com/science-lab/superresolution-microscopy-gives-new-insights-into-nuclear-porecomplex-organization/

BioTechniques 57:217 (October 2014) doi 10.2144/000114221 